\title{
HALF-QUADRATIC REGULARIZATION, PRECONDITIONING AND APPLICATIONS
}

\author{
Michael $\mathrm{Ng}^{*}$ \\ Department of Mathematics \\ The University of Hong Kong \\ Pokfulam Road, Hong Kong
}

\begin{abstract}
In this paper, we address a wide class of image deconvolution or reconstruction situations where a sought image is recovered from degraded observed image. The sought solution is defined to be the minimizer of an objective function combining a data-fidelity term and a edge-preserving, convex regularization term. Our objective is to speed up the calculation of the solution in a wide range of situations. We propose a method applying pertinent preconditioning to an adapted half-quadratic equivalent form of the objective function. The optimal solution is then found using an alternating minimization (AM) scheme. We focus specifically on Huber regularization. We exhibit the possibility get very fast calculations while preserving the edges in the solution. Preliminary numerical results are reported to illustrate the effectiveness of our method.
\end{abstract}

\section{INTRODUCTION}

This work addresses a wide class of image reconstruction situations where a sought image $\hat{x} \in R^{p}$ is recovered from degraded data $y \in R^{q}$ by minimizing an objective function $J: R^{p} \rightarrow R$ combining a data-fidelity term and a regularization term:

$$
\begin{aligned}
\hat{x} & =\min _{x \in R^{p}} J(x) \\
J(x) & =\|A x-y\|^{2}+\beta \sum_{i=1}^{r} \phi\left(d_{i}^{T} x\right) .
\end{aligned}
$$

In the expression above, $A \in R^{q \times p}$ represents the observation system and $\beta>0$ is a parameter. The regularization term involves a $\mathcal{C}^{1}$ convex function $\phi: R \rightarrow R$, applied to a set of linear transforms of the image $d_{i}^{T} x$ which are typically first or second-order differences between neighboring pixels. Such reconstruction methods are well-known to allow the obtention of high quality image estimates $\hat{x}$ when $\phi(t)$ approaches affine function when $|t| \rightarrow \infty[4,1,6]$.

* Research supported in part by Hong Kong Research Grants Council Grant Nos. HKU 7147/99P and HKU 7132/00P, and HKU CRCG Grant Nos. 10203408 and 10203501.
However, their use in different image reconstruction and image restoration applications can be practically limited by the numerical cost needed for the calculation of the estimate. The latter is usually calculated using iterative descent algorithms, based on the gradient of $J$. The calculation speed is mainly limited by the fact that the gradient of $J$ is non-linear and the Hessian matrices are usually illconditioned at each iteration. In this paper, we consider a a fast algorithm for the calculation of $\hat{x}$ by using a halfquadratic equivalent form of $J$ combined with pertinent preconditioning.

\section{TWO FORMS OF HALF-QUADRATIC REGULARIZATION}

Since [10] and [11], numerous algorithms have been proposed where $\hat{x}$ is calculated by minimizing an augmented objective function $G: R^{p} \times R^{r} \mapsto R$ which involves an auxiliary variable $s \in R^{r}$ :

$$
G(x, s)=\|A x-y\|^{2}+\beta \sum_{i=1}^{r} Q\left(d_{i}^{T} x, s_{i}\right)+\beta \sum_{i=1}^{r} \psi\left(s_{i}\right)
$$

where for every $s_{i} \in R$ the function $Q\left(., s_{i}\right): R \mapsto R$ is quadratic. The equivalence with $(1-2)$ is ensured by the requirement that for every $x \in R^{p}$,

$$
J(x)=\min _{s \in R^{r}} G(x, s) .
$$

The advantage of such an equivalent formulation is twofold: for every $s$ fixed, the gradient of $x \mapsto G(x, s)$ is an affine function whereas the non-quadratic part of $G$, that is $s \mapsto$ $G(x, s)$, is separable in $s$ and hence easy to minimize in a parallel way.

Some researchers have proposed and developed quadratic terms of the form $[2,6,12,8]$ :

$$
Q\left(d_{i}^{T} x, s_{i}\right)=\left(d_{i}^{T} x\right)^{2} s_{i} .
$$

A slightly different form for $Q$ has been proposed in [11] to perform stochastic optimization for a specific concave regularization:

$$
Q\left(d_{i}^{T} x, s_{i}\right)=\left(d_{i}^{T} x-s_{i}\right)^{2}
$$


Several authors generalized this approach $[7,1]$. Besides, the numerical effects relevant to these formulations have never been examined.

Recall that the numerical efficiency of the minimization of $G$ is tightly connected with the structure of its Hessian at each iteration. We observe that under (3); the Hessian of $G(., s)$ is

$$
2 A^{T} A+\beta D^{T} \operatorname{diag}(s) D
$$

where $D$ denotes the $p \times r$ matrix yielding $[D x]_{i}=d_{i} x$ for all $i$ and $x$ and $\operatorname{diag}(s)$ is a diagonal matrix with diagonal elements $s_{i}$. A drawback of this formulation is that the conditioning of $G(., s)$ depends on the value of $s$. In comparison, the Hessian of $G(., s)$ under (4) reads

$$
H:=2 A^{T} A+\beta D^{T} D
$$

hence it is independent of $s$. This is an important advantage which pushes us to focus on (4).

\section{PROPOSED ALGORITHM}

In our context, $G$ is convex function with respect to $(x, s)$. Therefore, with an initial guess $\left(x^{(0)}, s^{(0)}\right)$ for $(x, s)$, we can minimize $G(x, s)$ by first solving

$$
G\left(x^{(0)}, s^{(1)}\right) \equiv \min _{s} G\left(x^{(0)}, \cdot\right)
$$

and then

$$
G\left(x^{(1)}, s^{(1)}\right) \equiv \min _{x} G\left(\cdot, s^{(1)}\right) .
$$

We develop an alternating minimization (AM) algorithm in which the function value $G\left(x^{(n)}, s^{(n)}\right)$ always decreases as $n$ increases. More precisely, the algorithm is stated as follows:

Assume we have $x^{(k)}$ and $s^{(k)}$ :

- Solve for $s^{(k+1)}=\left[s_{1}^{(k+1)}, s_{2}^{(k+1)}, \cdots s_{r}^{(k+1)}\right]^{T}$ where $s_{i}^{(k+1)}$ can be determined by solving

$$
\min _{s_{i}^{(k+1)}} \psi\left(s_{i}^{(k+1)}\right)+\left(d_{i}^{T} x^{(k)}-s_{i}^{(k+1)}\right)^{2} .
$$

This minimization is very easy since it is performed for each $i$ separately. Notice that the next step does not involve the minimum value $\psi\left(s_{i}^{(k+1)}\right)$ but only its argument $s_{i}^{(k+1)}$

- Solve for $x^{(k+1)}$

$$
H x^{(k+1)}=2 A^{T} y+\sum_{i=1}^{r} s_{i}^{(k+1)} d_{i} .
$$

We remark that the matrix in the left side above is $H$ given in (6) and it is constant, whereas the right-side is easily updated at each iteration. The costly stage in the algorithm is the inversion of $H$. To this end, we propose to use pertinent preconditioning of $H$ in order to speed up the computation of $x^{(k+1)}$.

\section{PRECONDITIONING OF $G$}

Usually, image reconstruction problems are to recover $x$ from $y$ and the corresponding system matrix $A$. In these applications, these system matrices are Toeplitz-like, [13]. In this paper, we will consider the preconditioned conjugate gradient method to solve the linear system in (8) with Toeplitz-like coefficient matrix. Optimal transformed based matrices are used to precondition Toeplitz-like matrices in conjugate gradient iterations [9]. Part of their motivation was to exploit the fast inversion of transform based matrices. Numerical results suggest that the method converges very fast for a wide range of Toeplitz-like observation operators $A$.

\subsection{Circulant Preconditioners}

For a $p$-by- $p$ matrix $B$, the optimal circulant preconditioner $c_{F}(B)$ is defined to be the minimizer of $\|B-C\|_{F}$ over all $p$-by- $p$ circulant matrices $C$; see for instance [9]. Here $\|\cdot\|_{F}$ denotes the Frobenius norm. In each iteration, we solve the preconditioned system:

$$
\begin{aligned}
& \left(2 c_{F}(A)^{T} c_{F}(A)+\beta c_{F}\left(D^{T} D\right)\right)^{-1} H x^{(k+1)} \\
= & \left(2 c_{F}(A)^{T} c_{F}(A)+\beta c_{F}\left(D^{T} D\right)\right)^{-1} . \\
& \cdot\left[2 A^{T} y+\sum_{i=1}^{r} s_{i}^{(k+1)} d_{i}\right] .
\end{aligned}
$$

The main cost per iteration is the two matrix-vector multiplications: $\left(2 c_{F}(A)^{T} c_{F}(A)+\beta c_{F}\left(D^{T} D\right)\right)^{-1} v$ and $\left(2 A^{T} A+\right.$ $\left.\beta D^{T} D\right) w$. Since circulant matrices can be diagonalized by discrete Fourier matrices $F$, and hence the inversion of $p$-by- $p$ circulant systems can be done in $O(p \log p)$ operations. The cost of computing $\left(2 A^{T} A+\beta D^{T} D\right) w$ can be computed by embedding $A$ into a larger circulant matrix and then carrying out the multiplication. The multiplication thus requires $O(p \log p)$ operations [9].

\subsection{Cosine Transform Based Preconditioners}

Besides circulant preconditioners for Toeplitz-like blurring matrices, we note that discrete cosine transform matrices can diagonalized blurring matrices from symmetric blurring functions. For nonsymmetric blurring functions, we define the optimal cosine transform preconditioners $c_{C}(B)$ to be the minimizer of $\|B-Q\|_{F}$ over all $Q$ that can be diagonalized by cosine transform matrices. The preconditioners can speed up the convergence of iterative methods.

In view of the results in [13], it is easy to find the optimal cosine transform preconditioners for blurring matrices generated by nonsymmetric blurring functions. We just take the symmetric part of the blurring functions and form the (block) Toeplitz-plus-Hankel matrices. It has been shown theoretically and numerically that if the blurring function 
is close to symmetric, then the cosine transform preconditioner $c_{C}(A)$ will be a good approximation (hence a good preconditioner) to $A$.

\section{APPLICATIONS: IMAGE RECONSTRUCTION}

In the experiments, we consider reconstructing high resolution images from multiple under-sampled, shifted, degraded frames with sub-pixel displacement errors [3]. The resulting blurring matrices are spatially variant [3]. The classical Tikhonov regularization and the Neumann boundary condition are used in the reconstruction process. The preconditioners are derived by taking the cosine transform approximation of the blurring matrices. Here we briefly describe the model in high resolution image reconstruction. Details can be found in [3]. Consider a sensor array with $L_{1} \times L_{2}$ sensors in which each sensor has $N_{1} \times N_{2}$ sensing elements (pixels) and the size of each sensing element is $T_{1} \times T_{1}$. Our aim is to reconstruct an image of resolution $M_{1} \times M_{2}$, where $M_{1}=L_{1} \times N_{1}$ and $M_{2}=L_{2} \times N_{2}$. In order to have enough information to resolve the high-resolution image, there are sub-pixel displacements between sensors. In the ideal case, the sensors are shifted from each other by a value proportional to $T_{1} / L \times T_{2} / L$. However, in practice there can be small perturbations around these ideal sub-pixel locations due to imperfections of the mechanical imaging system. Thus, for $l_{1}, l_{2}=0,1, \cdots, L-1$ with $\left(l_{1}, l_{2}\right) \neq(0,0)$, the horizontal and vertical displacements $d_{l_{1} l_{2}}^{x}$ and $d_{l_{1} l_{2}}^{y}$ of the $\left[l_{1}, l_{2}\right]$-th sensor with respect to the $[0,0]$-th reference sensor are given by

$$
d_{l_{1} l_{2}}^{x}=\frac{T_{1}}{L}\left(l_{1}+\epsilon_{l_{1} l_{2}}^{x}\right) \quad \text { and } \quad d_{l_{1} l_{2}}^{y}=\frac{T_{2}}{L}\left(l_{2}+\epsilon_{l_{1} l_{2}}^{y}\right) .
$$

Here $\epsilon_{l_{1} l_{2}}^{x}$ and $\epsilon_{l_{1} l_{2}}^{y}$ denote respectively the normalized horizontal and vertical displacement errors. Let $f$ be the original scene. Then the observed low-resolution image $g_{l_{1} l_{2}}$ from the $\left(l_{1}, l_{2}\right)$-th sensor is modeled by:

$$
\begin{aligned}
& g_{l_{1} l_{2}}\left[n_{1}, n_{2}\right] \\
= & \int_{T_{2}\left(n_{2}-\frac{1}{2}\right)+d_{l_{1} l_{2}}^{y}}^{T_{2}\left(n_{2}+\frac{1}{2}\right)+d_{l_{1} l_{2}}^{y}} \int_{T_{1}\left(n_{1}-\frac{1}{2}\right)+d_{l_{1} l_{2}}^{x}}^{T_{1}\left(n_{1}+\frac{1}{2}\right)+d_{l_{1} l_{2}}^{x}} f\left(x_{1}, x_{2}\right) d x_{1} d x_{2} \\
+ & +\eta_{l_{1} l_{2}}\left[n_{1}, n_{2}\right] .
\end{aligned}
$$

for $n_{1}=1, \ldots, N_{1}$ and $n_{2}=1, \ldots, N_{2}$. Here $d_{l_{1} l_{2}}^{x}$ and $d_{l_{1} l_{2}}^{y}$ of the $\left[l_{1}, l_{2}\right]$-th sensor with respect to the $[0,0]$-th reference sensor are the horizontal and vertical displacements, and $\eta_{l_{1} l_{2}}$ is the noise corresponding to the $\left(l_{1}, l_{2}\right)$-th sensor. We interlace the low-resolution images to form an $M_{1} \times M_{2}$ image by assigning

$$
g\left[L\left(n_{1}-1\right)+l_{1} ; L\left(n_{2}-1\right)+l_{2}\right]=g_{l_{1} l_{2}}\left[n_{1}, n_{2}\right] .
$$

Here $g$ is an $M_{1} \times M_{2}$ image and is called the observed highresolution image. The blurring matrix corresponding to the

\begin{tabular}{c|c||c}
$\beta$ & $\alpha$ & Iter. \\
\hline $1 \mathrm{e}-2$ & $(5,10,20,40)$ & $(20,14,9,5)$ \\
$1 \mathrm{e}-3$ & $(5,10,20,40)$ & $(20,15,9,5)$ \\
$1 \mathrm{e}-4$ & $(5,10,20,40)$ & $(28,16,9,5)$
\end{tabular}

Table 1. Number of $A M$ iterations for $S N R=40 d B$.

\begin{tabular}{c|c||c}
$\beta$ & $\alpha$ & Iter. \\
\hline le-2 & $(5,10,20,40)$ & $(20,15,9,5)$ \\
le-3 & $(5,10,20,40)$ & $(17,13,9,5)$ \\
le-4 & $(5,10,20,40)$ & $(15,11,8,5)$
\end{tabular}

Table 2. Number of AM iterations for $\mathrm{SNR}=50 \mathrm{~dB}$.

$\left(l_{1}, l_{2}\right)$-th sensor under the Neumann boundary condition is given by

$$
H_{l_{1} l_{2}}\left(\epsilon_{l_{1}, l_{2}}\right)=H_{l_{1} l_{2}}^{x}\left(\epsilon_{l_{1}, l_{2}}^{x}\right) \otimes H_{l_{1} l_{2}}^{y}\left(\epsilon_{l_{1}, l_{2}}^{y}\right) .
$$

The blurring matrix for the whole sensor array is made up of blurring matrices from each sensor. The resulting blurring matrices are spatially variant. However, we remark the optimal cosine transform based preconditioners are very good approximation for these blurring matrices.

\section{NUMERICAL RESULTS}

In the tests, we concentrate on Huber regularizations which are defined using

$$
\phi(t)=\left\{\begin{array}{ccc}
\frac{1}{2} t^{2} & \text { if } & |t| \leq \alpha \\
\alpha|t|^{-}-\frac{1}{2} \alpha^{2} & \text { if } & |t|>\alpha
\end{array}\right.
$$

The threshold parameter $\alpha$ controls the size of the discontinuities modeled by the prior by providing a less severe edge penalty. The stopping criteria of the alternating minimization (AM) method and the preconditioned conjugate gradient (PCG) method are $\left\|x^{(k)}-x^{(k-1)}\right\|_{2}<20$ and $\left\|r^{(j)}\right\| /\left\|r^{(0)}\right\|<10^{-6}$ respectively, where $r^{(j)}$ is the normal equations residual after $j$ iterations.

Using the image reconstruction application in Section 5 , we first show the efficiency of our method. The number of AM iterations are listed in Tables 1 and 2. When $\alpha$ increases, the number of AM iterations decreases. However, when $\beta$ changes, the numbers of AM iterations are almost the same for different $\alpha$.

We also note that the numbers of iterations of using the PCG method for inverting the matrix in (6) are almost the same for each AM iteration. In Tables 3 and 4, the total numbers of $\mathrm{CG}$ and PCG iterations required to solve the linear systems in each AM step are listed. Here we used the optimal cosine transform based preconditioners for this image reconstruction problem. We see from the tables that it takes significantly more iterations using the CG method that those using the PCG method. 


\begin{tabular}{c||c|c}
$\beta$ & CG & PCG \\
\hline le-2 & $(480,336,216,120)$ & $(120,84,54,30)$ \\
le-3 & $(1180,885,531,295)$ & $(140,105,63,35)$ \\
1e-4 & $(4172,2384,1341,745)$ & $(252,144,81,45)$
\end{tabular}

Table 3. Number of CG iterations for solving the system with the coefficient matrix in (6) when $\mathrm{SNR}=40 \mathrm{~dB}$ for $\alpha=$ $(5,10,20,40)$.

\begin{tabular}{c||c|c}
$\beta$ & CG & PCG \\
\hline le-2 & $(480,360,216,120)$ & $(120,84,54,30)$ \\
le-3 & $(969,741,513,285)$ & $(140,105,63,35)$ \\
le-4 & $(1995,1463,1064,665)$ & $(252,144,81,45)$
\end{tabular}

Table 4. Number of CG iterations for solving the system with the coefficient matrix in (6) when $\mathrm{SNR}=50 \mathrm{~dB}$ for $\alpha=$ $(5,10,20,40)$.

Next we compare the number of iterations required to solve the linear systems with the coefficient matrices in (5) and (6). We find that the number of iterations of using CG or PCG for solving the linear system with the coefficient matrix in (5) is in average more than 300 . This number is much greater than those required for solving linear system with the coefficient matrix in (6). This demonstrates the effectiveness of our new formulation.

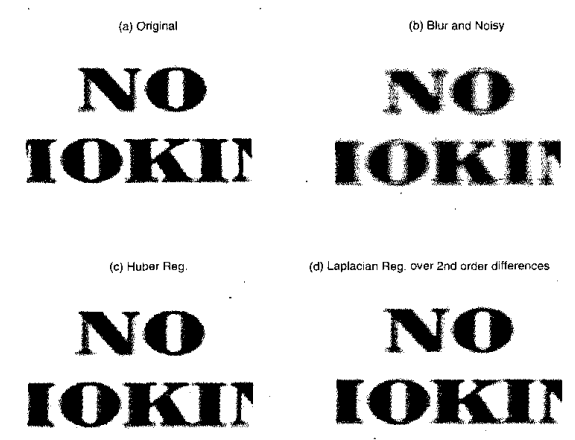

Fig. 1. Results of the first test image.

Finally, in Figure 1, the part of the original image (a), its observed high-resolution (b) and reconstructed images using Huber (c) and Laplacian (d) regularizations are shown in Figure 1. It is clear that the image using the Huber regularization is much better reconstructed than that using the Laplacian regularization.

\section{REFERENCES}

[1] G. Aubert and L. Vese, "A variational method in image recovery," SIAM Journal on Numerical Analysis, vol. 34, no. 5, pp. 1948-1979, 1997.
[2] Michael Black and Anand Rangarajan, "On the unification of line processes, outlier rejection, and robust statistics with applications to early vision," International Journal of Computer Vision, vol. 19, no. 1, pp. 57-91, 1996.

[3] N. Bose and K. Boo, "High-resolution Image Reconstruction with Multisensors," International Journal of Imaging Systems and Technology, vol 9, pp. 294-304, 1998.

[4] C. Bouman and K. Sauer, "A generalized Gaussian image model for edge-preserving MAP estimation," IEEE Transactions on Image Processing, vol. IP-2, no. 3, pp. 296-310, July 1993.

[5] Ch. Bouman and K. Sauer, "A unified approach to statistical tomography using coordinate descent optimization," IEEE Transactions on Image Processing, vol. 5, no. 3, pp. 480-492, Mar. 1996.

[6] P. Charbonnier, Laure Blanc-Féraud, Gilles Aubert, and Michel Barlaud, "Deterministic edge-preserving regularization in computed imaging," IEEE Transactions on Image Processing, vol. 6, no. 2, pp. 298-311, Feb. 1997.

[7] L. D. Cohen, "Auxiliary variables and 2-step iterative algorithms in computer vision problems," Journal of Mathematical Imaging and Vision, vol. 6, pp. 59-83, 1996.

[8] A. H. Delanay and Y Bressler; "Globally convergent edge-preserving regularized reconstruction: an application to limited-angle tomography," IEEE Transactions on Image Processing, vol. 7, pp. 204-221, Feb. 1998.

[9] R. Chan and M. Ng, "Conjugate gradient methods for Toeplitz systems," SIAM Review, vol 38, pp. 427-482, 1996.

[10] Donald Geman and Georges Reynolds, "Constrained restoration and recovery of discontinuities," IEEE Transactions on Pattern Analysis and Machine Intelligence, vol. PAMI-14, no. 3, pp. 367-383, Mar. 1992.

[11] Donald Geman and Chengda Yang, "Nonlinear image recovery with half-quadratic regularization," IEEE Transactions on Image Processing, vol. IP-4, no. 7 , pp. 932-946, July 1995.

[12] P. Kornprobst, R. Deriche, and G. Aubert, "Image sequence analysis via partial differential equations," Journal of Mathematical Imaging and Vision, vol. 11, no. 1, pp. 5-26, Oct. 1999.

[13] M. Ng, R. Chan and W. Tang, "A fast algorithm for deblurring models with Neumann boundary conditions," SIAM J. Sci. Comput., vol 21 pp. 851-866, 1999. 